\title{
Reason for Exclusion from Statistics
}

National Cancer Institute

\section{Source}

National Cancer Institute. Reason for Exclusion from Statistics. NCI Thesaurus. Code C117057.

A rationale for excluding a particular data point or subject from the statistical analysis. 